\title{
Online Model Racing based on Extreme Performance
}

\author{
Tiantian Zhang, Michael Georgiopoulos and Georgios C. Anagnostopoulos \\ zhangtt@knights.ucf.edu, michaelg@ucf.edu and georgio@fit.edu
}

\begin{abstract}
Racing algorithms are often used for offline model selection, where models are compared in terms of their average performance over a collection of problems. In this paper, we present a new racing algorithm variant, Max-Race, which makes decisions based on the maximum performance of models. It is an online algorithm, whose goal is to optimally allocate computational resources in a portfolio of evolutionary algorithms, while solving a particular problem instance. It employs a hypothesis test based on extreme value theory in order to decide, which component algorithms to retire, while avoiding unnecessary computations. Experimental results confirm that Max-Race is able to identify the best individual with high precision and low computational overhead. When used as a scheme to select the best from a portfolio of algorithms, the results compare favorably to the ones of other popular algorithm portfolio approaches.
\end{abstract}

Keywords: Online Model Selection, Racing Algorithm, Extreme Value Theory, Algorithm Portfolio

\section{Introduction}

The concept of Racing Algorithms (RAs) was originally developed for model selection in machine learning. Models, broadly speaking, could refer to any algorithm, operator or learning strategy. Given an ensemble of potential models, an RA sequentially evaluates each candidate on a series of benchmark instances of a problem domain, and eliminates under-performers as soon as sufficient statistical evidence supporting their removal is accumulated. Computational resources can be devoted to exploiting promising candidates by eliminating inferior candidates as early as possible. RAs maintain a reasonable balance between model optimality and computational complexity. Furthermore, RAs have been used extensively for algorithm tuning, algorithm configuration, and hybrid algorithm development; see [3] for a pertinent exposition.

The first RA, introduced in [14] and referred to as Hoeffding Racing (HR), computes an error bound for each model using Hoeffding's inequality. The ensemble of candidate models are tested on a set of problem instances, one at a time. Next, Hoeffding's inequality is used to estimate the true error from observed errors for each candidate. A candidate is discarded once the confidence interval for its true error rises above at least one confidence interval of the remaining candidates. A parametric analogue of HR was presented in [16], namely BRACE. In BRACE, leave-one-out cross validation errors of memory-based learners are assumed to be random variables from Gaussian distributions. Bayesian statistics are employed to detect if two errors are significantly different. In [15], Bernstein's inequality was employed in the RA. Their method was shown to eliminate inferior models faster than the HR method, mainly due to the higher quality of the concentration inequality. The most popular RA is F-Race and its counterpart I/F-Race [3], which successfully transferred the idea of racing to the problem of selecting configurations of parameterized algorithms. In FRace, a Friedman test is adopted for testing equivalence of model performance before considering pair-wise comparisons. The most recent RA is S-Race [26], a multi-objective RA, which addresses the problem of multi-objective model selection. S-Race features a non-parametric sign test and it employs Holm's stepdown procedure to control the overall probability of making false rejections. The returned models are optimal in the sense of Pareto optimality. Some other examples of RA are summarized in [26]. 
RAs are commonly used for offline model selection. In this scenario, a subset of all available samples is chosen as a training set, and models are compared in accordance to their overall average performance on the training set. The models with the best average performance are identified by the RAs. This is a pure exploration problem, which is at risk of returning models perform poorly on previously-unseen samples. In a different scenario of solving any given problem instances, one would like to maximize the performance quality by automatically allocating most computational resources to the optimal model/solver during problem solving. This is a typical online model selection problem, for which, many algorithms have been developed and applied in a wide variety of algorithm-problem settings. In [13, 1], for instance, model selection is formulated as a Markov Decision Process, and reinforcement learning is used to find the optimal algorithm scheduling strategy. In [9, 6], multi-armed bandit algorithms are employed to find the optimal assignment of available computational resources. In online settings, the time allocation can be adapted by monitoring algorithm execution to meet a reasonable trade-off between exploration and exploitation.

Classic online model selection primarily aims at maximizing the expected cumulative or maximum rewards. However, in many practical applications involving noise-less objective functions, optimizing extrema is more pertinent or important. For example, in algorithm selection for solving a given NP-hard combinatorial optimization problem, it is highly advantageous to dynamically determine and exploit the candidate heuristics that yield the best final solution; in this case, extreme performance is the only meaningful measurement worth considering.

In this paper, an online RA, Max-Race, is introduced. To the best of our knowledge, it is the first online model selection algorithm aiming at optimizing the extreme performance quality by making the best possible use of available resources. The Max-Race algorithm performs online evaluation/racing, where model comparison and selection is performed as each problem instance is evaluated. In Max-Race, the intermediate performance of each candidate is evaluated on a regular basis, while solving the problem. The expected (or predicted) maximum performance is estimated by utilizing notions of extreme value theory. In particular, a pair-wise hypothesis test is developed to identify significant difference between extreme performances of two given models. Models with inferior predicted maximum performance are gradually eliminated from the race and computational resources are increasingly invested in exploiting more promising candidate models.

The need for Max-Race is motivated by problems such as computational resources assignment in a population-based algorithm portfolio (AP). Population-based AP is an approach to automatically synthesize an ensemble of different population-based algorithms. The main motivation behind them is to enhance the overall performance of the component algorithms on any problem instance in terms of solution quality. Research on APs has gained significant attention in recent years with the booming development of powerful Evolutionary Algorithms (EAs). Recent developments on this type of problem are [21], [17] and [23]. In [21], the authors describe A Multi-algorithm Genetically Adaptive Method for Single Objective Optimization (AMALGAM-SO), which implements a self-adaptive learning strategy that automatically tunes the number of offspring each constituent algorithm will contribute to each generation. In addition, a novel elitism selection scheme is employed for generating better and diverse offspring. In [17], the authors construct a populationbased algorithm portfolio (PAP), in which the constituent algorithms run in parallel with solution migration occurring at regular intervals. The share of available computational resources remains fixed. Additionally, in the AP framework proposed in [23], each algorithm runs independently without any information exchange. In each generation, however, only the algorithm with the best predicted performance in the nearest common future point is executed, while others are temporarily suspended. The prediction only depends on the past history of their performance.

Nevertheless, the existing AP approaches have certain limitations. First of all, not all combinations of algorithms perform equally well, as demonstrated via experimental results in [21, 17]. Inappropriate selection of constituent algorithms may hinder the optimization process considerably. Secondly, it is not known how to synchronize the constituent algorithms so that their performances complement each other. For example, AMALGAM-SO incorporates elitism selection and premature convergence detection along with other mechanisms to better cooperate its component algorithms. Thirdly, considerable computation time is spent on constituents with inferior performance.

In this paper, we adopt Max-Race to construct a new population-based AP approach, named MaxRace Portfolio (MRP), which follows a simple Winner-Take-All (WTA) strategy. In MRP, the algorithms in the portfolio run concurrently without any information exchange. Max-Race employs an appropriate performance measure to eliminate algorithms producing inferior solutions. The proposed methodology frees 
practitioners from the need to know the constituent algorithms well enough to be able to effectively combine them. It also limits waste of computational effort on under-performing algorithms. MRP is similar to the one proposed in [25], in the sense that it aborts all except one algorithm based on the best objective value obtained after a specific number of function evaluations (NFEs).

The details of Max-Race and MRP, along with a brief description of Extreme Value Theory (EVT) related concepts, are provided in Section 2. In Section 3.1, the efficiency of Max-Race is analyzed from two aspects: the prediction accuracy and achieved computational savings compared to a Brute Forth Approach (BFA), which simply executes all the constituent algorithms to completion. Finally, in Section 3.2, the performance of MRP, consisting of PSO, CPSO, jDE, JADE, SaDE and CMA-ES solvers, is compared to two baseline APs (RandEA and BestEA) and three other state-of-the-art APs (AMALGAM-SO, PAP and MultiEA) for function optimization problems. The experimental results demonstrated that Max-Race is capable of identifying the best algorithms with high likelihood and by incurring low computational cost, when exploring for models, and, hence, maximizing the quality of the final solution by making the best possible use of available resources.

\section{Max-Race Description}

In this paper we propose a new RA variant, named Max-Race. Max-Race is an online RA, which identifies superior models and abandons poor performing ones as the models are evaluated against each problem instance. Under certain assumptions to be elaborated on later, Max-Race tries to retain only models that achieve the best final objective value. Subsequently, Max-Race is then utilized as part of a populationbased algorithm portfolio, called MRP. Without loss of generality, in this paper we regard all optimization problems as maximization problems; minimization problems can be addressed as well by maximizing the negative of an objective function.

\subsection{Extreme Value Theory Concepts}

The outcome of a stochastic optimizer can be viewed as an estimator of the optimum value of the objective function and, hence, is a random variable, typically, of unknown distribution. We are interested in the extreme values of this distribution as a method of assessing the peak performance of the optimizer. EVT is concerned with statistics of extreme or rare events [7]. Note that EVT concepts have been already used to study the distribution of outcomes acquired by stochastic optimizers in $[12,5,6]$.

A focal result of EVT pertaining to sample maxima is analogous to that of the Central Limit Theorem and sample means. As stated in [7], the sample maximum distribution of a series of i.i.d. (independent and identically distributed) samples, after appropriate normalization, converges to a Generalized Extreme Value (GEV) distribution as the sample size approaches infinity. Let $Y=\max _{i=1, \ldots, n} X_{i}$, where $X_{i}$ is a sequence of i.i.d. random variables with a common Cumulative Distribution Function (CDF) $H$, then we have

$$
\lim _{n \rightarrow \infty} \operatorname{Pr}\left\{\frac{Y-b_{n}}{a_{n}} \leq y\right\}=\lim _{n \rightarrow \infty} H^{n}\left(a_{n} y+b_{n}\right)=G(y)
$$

where $a_{n}>0$ and $b_{n}$ are normalization coefficients and the asymptotic CDF $G(y)$ has the form

$$
G(y)=\exp \left\{-\left[1+\tilde{\xi}\left(\frac{y-\tilde{\mu}}{\tilde{\sigma}}\right)\right]^{-1 / \tilde{\xi}}\right\}
$$

The aforementioned CDF corresponds to the so called GEV distribution with location parameter $\tilde{\mu}$, scale parameter $\tilde{\sigma}>0$ and shape parameter $\tilde{\xi}$. Note that, depending on the value of its parameters, it specializes to one of three types of distributions: Fréchet, Weibull and Gumbel [7].

Based on Equation (1), it can be shown that, in the limit, the tail distribution of the $X_{i}$ 's tends to a three-parameter CDF as shown below

$$
\lim _{u \rightarrow u^{*}} \operatorname{Pr}\{X>x \mid X>u\}=F_{\xi, \mu, \sigma}(x)
$$


where $u^{*}$ is the endpoint of distribution $H$ and

$$
F_{\xi, \mu, \sigma}(x)=\left\{\begin{array}{cc}
1-\left(1+\frac{\xi(x-\mu)}{\sigma}\right)^{-1 / \xi} & \text { for } \xi \neq 0 \\
1-\exp \left(-\frac{x-\mu}{\sigma}\right) & \text { for } \xi=0
\end{array}\right.
$$

is called the Generalized Pareto Distribution (GPD), where $\sigma>0$, and $x \geq \mu$, when $\xi \geq 0$, and $\mu \leq x \leq$ $\mu-\sigma / \xi$, if otherwise. The three parameters $\xi, \mu, \sigma$ are referred to as shape, location and scale parameters, respectively and are uniquely determined by $\tilde{\xi}, \tilde{\mu}, \tilde{\sigma}$ of the associated GEV. Similar to the GEV distribution, the GPD contains three distribution families: when $\xi>0$, we have an ordinary Pareto distribution; when $\xi=0$, we have an exponential distribution; and when $\xi<0$, a short-tailed Pareto II distribution. Finally, note that the Probability Distribution Function (PDF) of the GPD is derived as

$$
f_{\xi, \mu, \sigma}(x)=\frac{1}{\sigma}\left(1+\frac{\xi(x-\mu)}{\sigma}\right)^{-\frac{1}{\xi}-1}
$$

Correspondingly, there are two basic approaches to modeling the parametric distribution of extreme values in EVT: Block Maxima (BM) and Point Over Threshold (POT). BM is the most classical approach in EVT. In BM, the data are collected in chunks (blocks) of equal size. The distribution of the maximum of each block follows approximately a GEV distribution. However, the BM approach doesn't make efficient use of the data, since only the sample maxima of large blocks are utilized. The POT approach may utilize data better, because all observations exceeding a sufficiently high threshold are used towards GPD parameter estimation. Moreover, due to the "threshold stability" property ${ }^{1}$ of GPD, methods based on POT are generally known to be more powerful for practical applications, such as ones in the areas of insurance, finance and environmental studies.

\subsection{Modeling Model Performance via GPDs}

The models considered in this paper are Evolutionary Computation (EC) algorithms, but our approach is applicable to a more general notion of what is considered as a model. EAs are population-based algorithms, where the population is a collection of samples in the decision space; these samples are updated iteratively through a guided random search. For a specific optimization problem with a bounded (global) maximum, such algorithms aim to produce a maximizer, which, hopefully, will yield the problem's global maximum. Due to the stochastic nature of EAs, the optimization outcomes could be described by their distribution. Since we are only interested in extreme outcomes, such as maxima, the intermediate outcomes can be regarded as samples along the right tail of the underlying distribution. In this framework, the POT approach is a natural choice to model the distribution of algorithm performance. In particular, the GPD approximation will be more accurate as evolution continues, because the underlying sampling distribution tends to become more stable as more samples gather close to the right endpoint. Our reported simulation results also support this thesis.

Let gbest $_{t}$ refer to the best objective value obtained so far (by time/iteration) $t$ of an EA. In essence, gbest $_{t}$ is a sequence of random variable of a discrete time Markov chain since gbest $_{t} \triangleq \max \left\{\right.$ pop $_{t}$, gbest $\left._{t-1}\right\}$, where pop $_{t}$ are samples of generation $t$. We assume that after some mixing time $t_{0}$, the Markov chain enters a state of equilibrium, where the collected $\left\{g_{b e s t_{t}}\right\}$ with $t>t_{0}$ can be regarded as i.i.d from a stationary distribution. Therefore, we will conceptually limit ourselves to EAs with the property that the stochastic sequence $\left\{g b e s t_{t}\right\}_{t=1}^{\infty}$ has a sub-sequence $\left\{g b e s t_{t}\right\}_{t=t_{o}}^{\infty}$ for very large $t_{o}$, such that the aforementioned subsequence contains i.i.d. samples. Such an EA evolves solutions and, after the passage of many generations, its sampling distribution tends to be stationary, even if in an approximate sense.

Along this rationale, we identify gbest $t_{t}$ after every generation, and, due to our i.i.d. assumption, we model the upper order statistics of the obtained sample by a GPD. In particular, we only consider the special case of GPD with $\xi<0$, which has a right endpoint $\mu-\sigma / \xi$.

Figure 1 shows the merits of following a POT approach in a practical setting. The displayed data is collected from one run of CPSO [20] in the context of a multi-modal function optimization problem.

\footnotetext{
${ }^{1}$ If the upper statistics of $X$ follow a GPD for a fixed threshold level $u$, the conditional distribution of $X>v$ for any higher threshold $v>u$ also follows a GPD with the same $\xi$ but a shifted $\sigma$.
} 
However, in the POT framework, the selection of an appropriate threshold $u$ is crucial before fitting, which involves balancing the bias and the variance of parameter estimators. For example, the GPD fit in Figure 1c is not as good as the one in Figure 1b because its $u$, which equals to the q-quantile of $s$ samples, is too large.

Note that several threshold selection methods are proposed in the literature [19]. However, we'll entertain fixed quantile rules as a simple choice that is justified by the threshold stability property of the GPD; specifically, the $q$-quantile of the sample is chosen as the threshold. The determination of a proper $q$ value is closely related to the $t_{0}$ value in the previous discussion. As the algorithm progresses, we expect more objective values to closely cluster below the endpoint. Note that $g_{b e s t_{t}}$ is a non-decreasing process with a fixed $t_{0}$. Accordingly, in our setting, the $q$ value decreases exponentially with the sample size $s$, viz. $q(s)=q_{0} e^{-\lambda s}$. Moreover, the quantity $q_{0}$ is chosen to be smaller than 0.9 , a typical value used in POT applications, since the sample values are already maxima of some optimization process. $q_{0}$ and $\lambda$ are determined empirically, based on our experimental observations, so that $q_{100}=0.7, q_{5000}=0.01$. After determining the threshold, the Method of Moments (MOM) [8] is adopted for estimating the GPD parameters $\xi, \mu$ and $\sigma$. MOM is computationally straightforward and the estimates are asymptotically normal, when $\xi<0$. As shown in Figure 1a and Figure 1b, the GPD fit becomes increasingly better against the empirical distribution as evolution progresses, if the $u$ value is properly selected. Note that the data fits better in the case of negative skewness and large magnitudes.

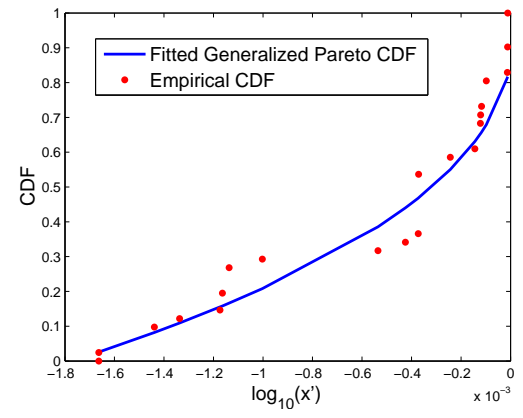

(a) $q=0.7, s=100$

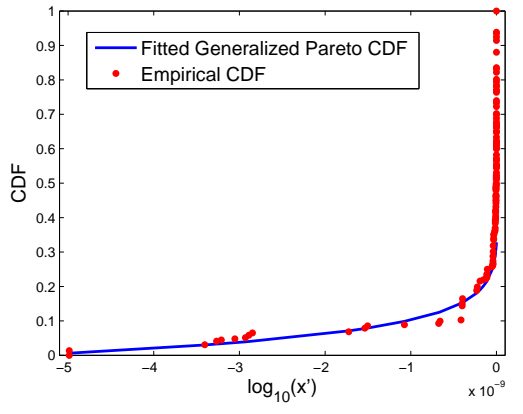

(b) $q=0.5, s=500$

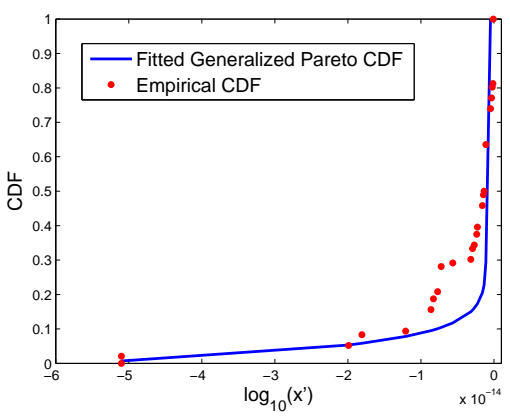

(c) $q=0.9, s=500$

Figure 1: GPD fit for gbest $_{t}$ collected from a CPSO run on a multi-modal function optimization problem. The $x^{\prime}$ shown below the $x$-axis represents the normalized value of $x$

\subsection{Max-Race Framework}

RAs for classic offline model selection compare models based on their average performance. In contrast to them, Max-Race tries to identify the model with the best extreme performance for online selection.

The framework of Max-Race is similar to a typical RA procedure. The Max-Race algorithm follows a suspend-and-resume scheme. It starts with an ensemble of models, which are run in parallel without information exchange. For each model, the intermediate best objective value obtained is periodically recorded. Repeatedly, execution is suspended and a statistical test is performed to assess the best maximum perfor- 
mance of each model based on historical performance. If sufficient statistical evidence shows, that a particular model is inferior (meaning that its best maximum performance is significantly worse than all other models), it is removed from the ensemble. Execution is subsequently resumed and the whole procedure is repeated until either a pre-specified time limit is exceeded or a single model remains.

\subsection{Inferring Inequivalence of Endpoints}

Max-Race employs a hypothesis test to detect differences between the sample maxima of two samples. The hypothesis test is parametric and assumes that the sampled data are i.i.d stemming from a 3-parameter GPD with an upper (finite) endpoint. Testing statistical difference between endpoints has received barely any attention in the literature, probably due to the lack of practical motivations so far. Therefore, for the purposes of our work, we develop one to identify such differences between the right endpoints of two GPDs.

Given the two sets of observations $X_{i}, i=1,2, \cdots, n$ from model $A$ and $Y_{j}, j=1,2, \cdots, m$ from model $B$, where $X_{i} \sim F_{\xi_{A}, \mu_{A}, \sigma_{A}}(x)$ and $Y_{j} \sim F_{\xi_{B}, \mu_{B}, \sigma_{B}}(y)$ with $\xi_{A}<0, \xi_{B}<0$, the null hypothesis is $H_{0}:$ $\mu_{A}-\frac{\sigma_{A}}{\xi_{A}}=\mu_{B}-\frac{\sigma_{B}}{\xi_{B}}$, indicating the equivalence of the endpoints. Correspondingly, the alternative hypothesis is $H_{a}: \mu_{A}-\frac{\sigma_{A}}{\xi_{A}}>\mu_{B}-\frac{\sigma_{B}}{\xi_{B}}$ assuming that the estimated endpoint for model $A$ is larger than the one of model $B$. The sample maxima of the two populations are used as estimates of the corresponding endpoints. Let $U \triangleq \max \left\{X_{1}, \cdots, X_{n}\right\}$, and $V \triangleq \max \left\{Y_{1}, \cdots, Y_{m}\right\}$. Based on our earlier stated assumptions, the PDFs of $U$ and $V$ are provided below:

$$
f_{U}(u)=n f_{\xi_{A}, \mu_{A}, \sigma_{A}}(u) F_{\xi_{A}, \mu_{A}, \sigma_{A}}^{n-1}(u)
$$

and

$$
f_{V}(v)=m f_{\xi_{B}, \mu_{B}, \sigma_{B}}(v) F_{\xi_{B}, \mu_{B}, \sigma_{B}}^{m-1}(v)
$$

The test statistic we consider is the difference $\Delta=U-V$ between the two sample maxima, whose PDF can be shown to be

$$
f_{\Delta}(\delta)=\int_{\mu_{A}}^{\mu_{A}-\frac{\sigma_{A}}{\xi_{A}}} f_{U}(u) f_{V}(u-\delta) d u
$$

Since the range of $U$ is $\left[\mu_{A}, \mu_{A}-\frac{\sigma_{A}}{\xi_{A}}\right]$ and of $V$ is $\left[\mu_{B}, \mu_{B}-\frac{\sigma_{B}}{\xi_{B}}\right]$, the range of $\Delta$ will be $\left[\mu_{A}-\mu_{B}+\frac{\sigma_{B}}{\xi_{B}}, \mu_{A}-\right.$ $\left.\mu_{B}-\frac{\sigma_{A}}{\xi_{A}}\right]$. However, under $H_{0}$, the range of $\Delta$ is $\left[\frac{\sigma_{A}}{\xi_{A}},-\frac{\sigma_{B}}{\xi_{B}}\right]$. Therefore, the probability $P(\Delta \leq d)$ under $H_{0}$ is

$$
\begin{aligned}
& P(\Delta \leq d)=\int_{\frac{\sigma_{A}}{\xi_{A}}}^{d} f_{\Delta}(\delta) d \delta \\
& =\int_{0}^{1} F_{\xi_{B}, \mu_{B}, \sigma_{B}}^{m}\left\{\frac{\sigma_{A}\left[\left(1-P^{\frac{1}{n}}\right)^{-\xi_{A}}-1\right]}{\xi_{A}}+\mu_{A}-\frac{\sigma_{A}}{\xi_{A}}\right\} d P \\
& -\int_{0}^{1} F_{\xi_{B}, \mu_{B}, \sigma_{B}}^{m}\left\{\frac{\sigma_{A}\left[\left(1-P^{\frac{1}{n}}\right)^{-\xi_{A}}-1\right]}{\xi_{A}}+\mu_{A}-d\right\} d P
\end{aligned}
$$

where $P=F_{\xi_{A}, \mu_{A}, \sigma_{A}}^{n}(u)$. When observing a value of $d$ for the difference between the estimated maximum performance of model A and B, we can compute the $p$-value of the proposed test as follows

$$
\pi(d ; A, B)=P(\Delta>d)=1-P(\Delta \leq d)
$$

If $\pi(d ; A, B)$ is smaller than the predefined Type I error rate $\alpha, H_{0}$ will be rejected. Since there is no closed-form expression for Equation (9), the adaptive Gaussian quadrature method for numerical integration is used to approximate the $\pi(d ; A, B)$ value.

Note that the parameter values needed for calculating $\pi(d ; A, B)$ are estimated from the data. The values $\mu_{A}$ and $\mu_{B}$ are first estimated using MOM. After deriving the location parameters, the original data are shifted accordingly and the values of $\sigma_{A}, \xi_{A}, \sigma_{B}$, and $\xi_{B}$ under $H_{0}$ are estimated by a hybrid method 
consisting of Maximum Likelihood Estimation and Maximum-Goodness-of-Fit Estimation [22], since the Maximum Likelihood method is not applicable when $\xi<-1$. For a detailed description of this estimation approach, we refer the interested reader to [22].

\subsection{Algorithm Portfolio based on Max-Race}

The online racing framework can be easily extended to form an AP. In this subsection, we present a novel population-based AP approach hinging on Max-Race and referred to as MRP. After initialization, each candidate algorithm in MRP runs independently on its own population. For each sub-population, the gbest value is preserved for every generation. Once the number of new gbest values collected reaches $N_{c s}$, the component algorithms are compared, pairwise, using the test proposed in Section 2.4. Those with inferior maximum performance will be stopped once sufficient statistical evidence is presented. The portfolio execution stops when it reaches the maximal NFEs. No test is necessary if only one algorithm is left in the portfolio. Furthermore, as a multiple comparison problem, the Benjamini-Hochberg-Yekutieli procedure [2] is implemented to control the overall false discovery rate, denoted as $F D R(\cdot, \cdot)$ in Algorithm 1. It makes rejections based on given $p$-values and the predefined confidence level $\alpha$. The output is the set of indices corresponding to the models with inferior maximum performance. Since the GPD fit becomes more accurate as more data is collected, the $\alpha$ value increases at a rate proportional to the sample size, and is given by

$$
\alpha_{s}=\alpha_{0} e^{-\beta\left(s_{\max }-s\right)}
$$

where $s_{\max }$ is the largest possible sample size and $s$ is the current sample size. The values $\alpha_{0}$ and $\beta$ are chosen empirically such that $\alpha_{s_{\max }}=0.01$ and $\alpha_{s_{0}}=1 e-30$.

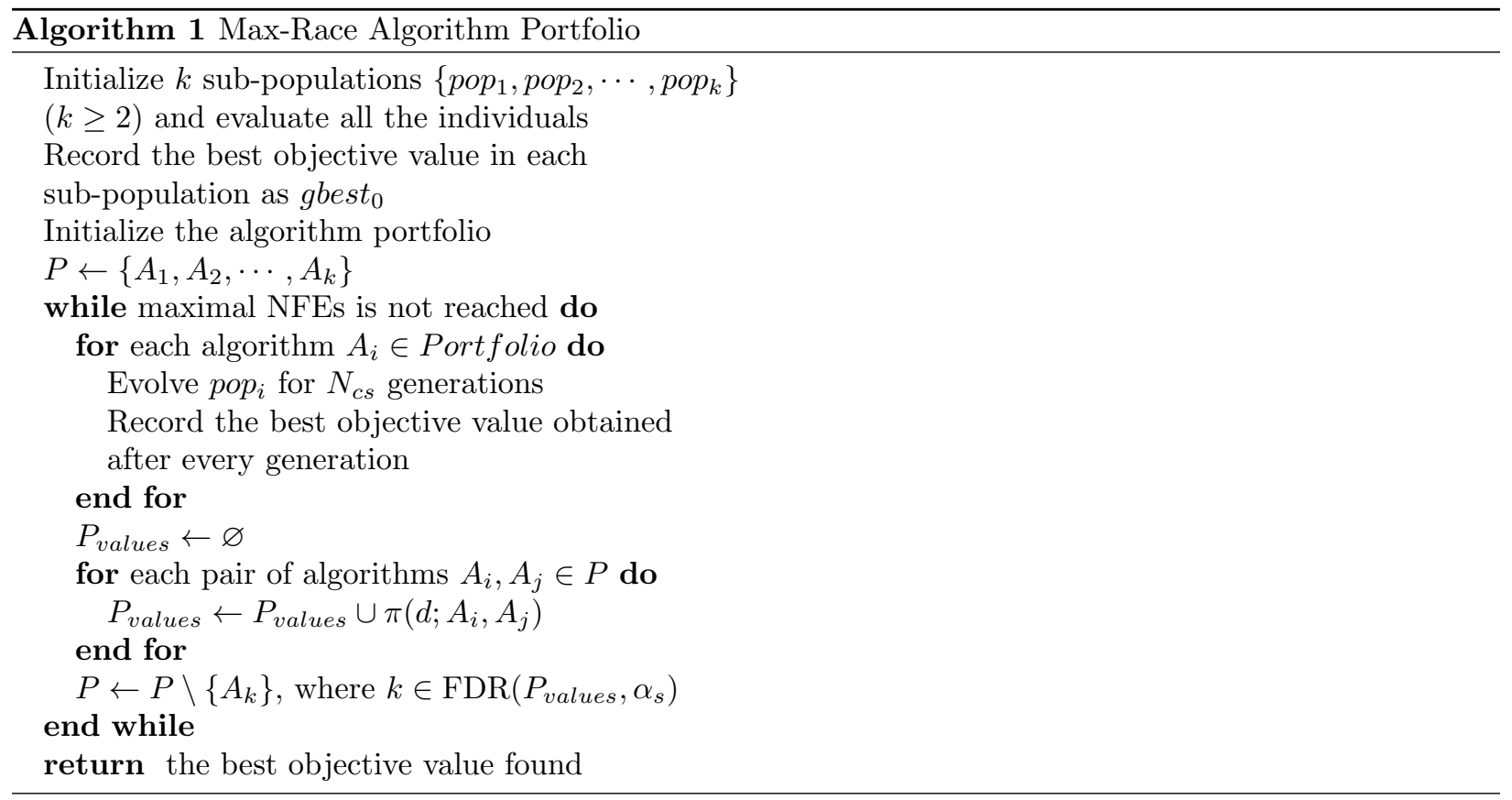

\section{Experimental Outcomes}

In this section, we assess the performance of Max-Race and its corresponding AP approach, MRP. The following suite of algorithms are used in the MRP design: PSO [27], CPSO [20], jDE [4], JADE [24], SaDE [18], and CMA-ES [11], which are popular EAs having demonstrated outstanding performance in the relevant literature. The test problems are multi-modal maximization problems with a bounded search space that are generated via a Gaussian landscape generator [10]. The boundary of the search space is set to be $[-100,100]^{D}$, where $D$ is the dimensionality of the problem. The advantage of Max-Race is illustrated in two 
aspects: the efficiency of Max-Race for online model selection and the competitiveness of MRP compared to other population-based APs.

\subsection{Max-Race Efficiency Analysis}

A simple, yet time-consuming, approach for finding the optimal algorithm is the BFA, which applies all the candidate algorithms on the problem of interest and runs them to completion. To demonstrate the efficiency of Max-Race, we compare it to BFA. Two performance metrics are specified: prediction accuracy, denoted by $P A$, and ratio of Max-Race to BFA running time to completion, denoted by $R T R$ (running time ratio). $P A$ is 1 if the algorithms with the true maximum performance are retained at the end of Max-Race execution, and 0 otherwise. $R T R$ is calculated as the ratio of the NFEs spent by Max-Race to the NFEs spent by BFA. Ideally, $P A$ should approach 1 and $R T R$ should approach 0 .

The efficiency of Max-Race is evaluated on 30 benchmark functions with dimensions $D=10$ and $D=30$. In each run, the constituent algorithms start with different sub-populations of size $5 D$. In BFA, each subpopulation evolves for $5000 \times 5 D$ NFEs, which is large enough to ensure convergence to a solution for a variety of benchmark functions and EAs. The intermediate gbest values from one run of BFA are collected as pseudo-samples for simulating a run of Max-Race. In Max-Race, the statistical comparison is performed every 10 generations $\left(N_{c s}=10\right)$. For each optimization task, the average $P A$ and $R T R$ values over 25 runs are calculated, as well as the average number of models with maximum performance in the BFA $\left(N_{b}\right)$ and the average number of models retained by the end of Max-Race $\left(N_{r}\right)$. However, due to space limitations, we only report a summary of them in Table 1 . As observed in Table 1, prediction accuracy is high, especially when $D=10$. In $D=30$, the task of identifying the best algorithm appears more challenging since the $P A$ values decrease on average. For high-dimensional and multi-modal objective functions, the algorithms show similar performance since it is more difficult to find a good solution (see Table 2). As a result, Max-Race cannot identify the real winner. Instead, it yields the algorithm with slightly less optimal performance. Such phenomenon is reasonable because Max-Race trades off some computational effort with prediction accuracy. However, the effect of problem dimension on the performance of Max-Race deserves future investigation. In terms of $R T R$ values, the computational burden for the Max-Race algorithm is always seen to be much lower than for the BFA algorithm.

Table 1: Summary of the comparison result between the BFA and Max-Race: $P A, R T R, N_{b}$ and $N_{r}$

\begin{tabular}{c|ccccc}
\hline Dim & & $P A$ & $R T R$ & $N_{b}$ & $N_{r}$ \\
\hline \multirow{4}{*}{10} & max & 1 & 0.30 & 3.92 & 1.24 \\
& median & 0.91 & 0.23 & 1.42 & 1.00 \\
& min & 0.80 & 0.22 & 1.04 & 1.00 \\
& std & 0.07 & 0.02 & 0.60 & 0.06 \\
\hline \multirow{4}{*}{30} & max & 1 & 0.46 & 2.44 & 2.20 \\
& median & 0.83 & 0.41 & 1.28 & 2.00 \\
& min & 0.36 & 0.38 & 1.00 & 1.92 \\
& std & 0.13 & 0.02 & 0.38 & 0.05 \\
\hline
\end{tabular}

Identifying the best algorithm is not necessarily the ultimate goal of online racing. Instead, the goal of Max-Race is to maximize the quality of the solution under time constraints. Therefore, we compare MRP with two baseline algorithms, namely a simple algorithm portfolio approach which randomly selects an algorithm (RandEA), and an ideal algorithm portfolio approach which always selects the best algorithm (BestEA), and we use the pseudo-samples generated from the previous experiments to do so. A maximum of $5000 \times 5 D$ NFEs is allowed for MRP, RandEA and BestEA. On the basis of a paired Wilcoxon signed-rank test with significance level 0.01, MRP is better than RandEA in all cases (i.e., a set of 25 runs considering a particular function to optimize). Meanwhile, BestEA only outperforms MRP significantly in $13 \%$ of the cases for $D=30$, as shown in Table 2 .

To better demonstrate how MRP performs compared to RandEA and BestEA, we estimate the density of their outcomes, 25 runs for each optimization task, from bootstrapped samples by using a kernel smoothing function. In Figure 2, we plot the density of means of the bootstrapped samples for a specific objective 
function $\left(f_{5}\right.$ with $\left.D=30\right)$. As shown, MRP accomplished nearly equal outcomes to those of BestEA and both displayed an advantage over RandEA.

\subsection{Comparisons of APs}

To evaluate the performance of MRP, we compare it to three population-based APs: AMALGAM-SO [21], PAP [17] and Multiple Evolutionary Algorithm (MultiEA) [23], with the same constituent algorithms. Another set of 20 problem instances are generated with $D=30$. The total population size is $5 D$, which is equally divided into sub-populations, when initialized. The maximum number of NFEs is set to $3 \times 10^{5}$, which is commonly used in the literature. Other AP specific parameters are set according to suggested values in the respective original papers.

In Table 3, we present the results of MRP, AMALGAM-SO, PAP and MultiEA for 20 problem instances. The typical measures of merit, mean and standard deviation of the final outcomes produced over 25 runs, are reported. In addition, the Wilcoxon signed-rank test with significance level 0.01 is adopted to identify significant difference in terms of performance. In Table 3, entries are underlined to highlight algorithms

Table 2: Mean values of final outcomes over 25 runs for MRP, BestEA, and RandEA; 30 problem instances for $D=30$, with the true global maximum value of each function. If the entry is bold-faced, the associated algorithm significantly outperforms MRP. If the entry is underlined, the associate algorithm is significantly outperformed by MRP.

\begin{tabular}{c|cccc}
\hline Probl. & MRP & BestEA & RandEA & $\begin{array}{c}\text { Global } \\
\text { Optimum }\end{array}$ \\
\hline$f_{1}$ & 168.39 & 171.09 & $\underline{107.01}$ & 389.68 \\
$f_{2}$ & 275.45 & 275.79 & $\underline{170.20}$ & 341.94 \\
$f_{3}$ & 108.04 & 114.57 & $\underline{81.88}$ & 193.99 \\
$f_{4}$ & 12.76 & $\mathbf{1 3 . 6 1}$ & $\underline{11.43}$ & 29.16 \\
$f_{5}$ & 161.79 & 165.23 & $\underline{69.91}$ & 193.99 \\
$f_{6}$ & 103.62 & 105.39 & $\underline{83.65}$ & 125.27 \\
$f_{7}$ & 41.19 & 41.20 & $\underline{36.25}$ & 287.79 \\
$f_{8}$ & 157.77 & 157.77 & $\underline{94.40}$ & 470.55 \\
$f_{9}$ & 7.76 & 8.46 & $\underline{5.99}$ & 9.93 \\
$f_{10}$ & 34.47 & 34.76 & $\underline{\underline{24.68}}$ & 41.61 \\
$f_{11}$ & 14.22 & 14.24 & $\underline{9.51}$ & 39.93 \\
$f_{12}$ & 187.78 & $\mathbf{2 2 4 . 3 6}$ & $\underline{83.48}$ & 275.21 \\
$f_{13}$ & 256.18 & 269.29 & $\underline{\underline{14.61}}$ & 415.96 \\
$f_{14}$ & 50.42 & 50.55 & $\underline{31.93}$ & 327.66 \\
$f_{15}$ & 78.82 & 89.61 & $\underline{\underline{48.50}}$ & 465.00 \\
$f_{16}$ & 62.32 & 68.56 & $\underline{39.70}$ & 400.16 \\
$f_{17}$ & 274.44 & 274.44 & $\underline{193.69}$ & 473.35 \\
$f_{18}$ & 30.03 & 35.38 & $\underline{\underline{16.73}}$ & 49.48 \\
$f_{19}$ & 13.43 & 13.59 & $\underline{9.56}$ & 70.55 \\
$f_{20}$ & 44.19 & 58.38 & $\underline{\underline{17.23}}$ & 49.48 \\
$f_{21}$ & 102.14 & 104.98 & $\underline{69.58}$ & 175.20 \\
$f_{22}$ & 257.90 & 262.40 & $\underline{\underline{131.12}}$ & 275.21 \\
$f_{23}$ & 155.67 & 157.51 & $\underline{\underline{103.49}}$ & 183.65 \\
$f_{24}$ & 6.65 & 6.91 & $\underline{3.91}$ & 316.59 \\
$f_{25}$ & 34.47 & 35.04 & $\underline{\underline{26.61}}$ & 41.61 \\
$f_{26}$ & 13.98 & 14.20 & $\underline{9.37}$ & 39.93 \\
$f_{27}$ & 178.67 & $\mathbf{2 2 0 . 1 7}$ & $\underline{61.14}$ & 341.94 \\
$f_{28}$ & 1.38 & 1.39 & $\underline{\underline{1.06}}$ & 10.63 \\
$f_{29}$ & 233.32 & $\mathbf{2 6 5 . 1 9}$ & $\underline{168.22}$ & 446.68 \\
$f_{30}$ & 30.75 & 32.21 & $\underline{19.86}$ & 73.57 \\
\hline & & & & \\
\hline
\end{tabular}




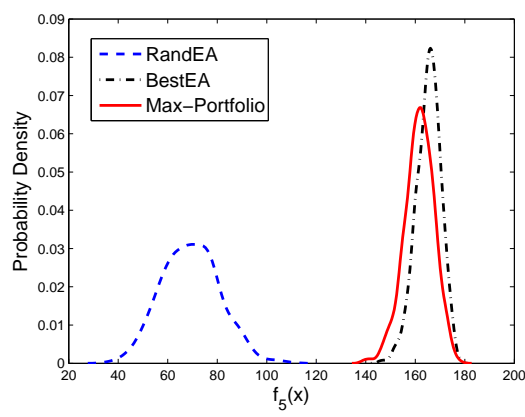

Figure 2: Bootstrap distributions of the means of final outcomes of MRP, RandEA and BestEA for $f_{5}$ with $D=30$

that are significantly outperformed by MRP. If MRP is significantly outperformed by an algorithm, the corresponding algorithm-specific entry is highlighted in boldface. As the results show, MRP does significantly better than AMALGAM-SO, PAP and MultiEA in 13, 11, and 13 out of 20 problem instances, while it is only significantly outperformed by them in 2,1 and 2 cases, respectively.

The overwhelming advantage of MRP confirms that applying Max-Race for online model selection is a very promising way of forming an algorithm portfolio. Given an optimization task, Max-Race is typically able to identify the ultimate winner from an ensemble of algorithms incurring much less computational overhead. MRP reflects a successful application of Max-Race, which makes the best use of computational resources for fully exploring and exploiting the search domain.

Meanwhile, the poor performance of AMALGAM-SO reveals that its success is highly dependent on synergy between the constituent algorithms. It requires a well-designed synchronization mechanism based on the characteristics of the constituent algorithms including population sharing, elitism selection, diversity control, premature convergence detection, etc. A user cannot blindly adopt the recommended settings in AMALGAM-SO's original paper for an arbitrary set of constituents, because the optimal combination of these operators needs careful tuning, which is computationally expensive.

Compared to AMALGAM-SO, PAP shows slightly better performance, since the sub-populations are only interleaved by some occasional migration of the best individual.

Among the three APs, MultiEA shares the most similar design principle as MRP: the sub-populations run independently without any interaction and only the algorithm with the best predicted performance is selected to run. However, its prediction is shortsighted in that the winner is chosen in terms of its estimated performance in the nearest common future point. And such estimation is based on bootstrapping samples collected after linear regression on the evolutionary curve. Therefore, it tends to squander time on algorithms that converge to some local maximum rapidly.

\section{Conclusions}

In this paper, an online Racing Algorithm (RA) is proposed, namely Max-Race. Max-Race tackles online model selection problem, aiming at optimizing the extreme performance quality by adaptively allocating available computational resources while solving a particular optimization problem instance. More specifically, Max-Race approximates the underlying distribution of model performance, measured as the best outcome obtained, via a Point Over Threshold (POT) approach rooted in Extreme Value Theory (EVT). A parametric hypothesis test based on the Generalized Pareto Distribution (GPD) is developed to identify significant difference between maximum performances of two models. The model with significant worse maximum performance will be discarded as early as possible.

The efficiency of Max-Race is evaluated by comparison to the Brute Forth Approach (BFA), a method which simply runs all the constituent algorithms to completion, for model selection. Max-Race is capable of identifying the best algorithm with high likelihood for a low computational cost. To better evaluate its performance, Max-Race is applied as a new algorithm portfolio (AP) approach, with 6 constituent algorithms: PSO, CPSO, jDE, JADE, SaDE and CMA-ES. The resulting AP, named Max-Race Portfolio (MRP), is 
compared to two baseline APs (RandEA and BestEA) and three other APs (AMALGAM-SO, PAP and MultiEA) on a collection of optimization problems. MRP's remarkable performance, that was experimentally demonstrated, implies that Max-Race is a promising online model selection algorithm.

Table 3: Mean and standard deviation (std) values of the final outcomes, out of the 25 runs, of MRP, AMALGAM-SO, PAP and MultiEA for 20 problem instances with $D=30$

\begin{tabular}{|c|c|c|c|c|c|}
\hline Probl. & & MRP & $\begin{array}{l}\text { AMALG } \\
\text { AM-SO }\end{array}$ & PAP & MultiEA \\
\hline \multirow{2}{*}{$f_{1}$} & mean & 53.77 & $\underline{43.00}$ & 52.05 & 42.20 \\
\hline & std & 2.27 & $\overline{4.21}$ & 4.41 & 3.76 \\
\hline \multirow{2}{*}{$f_{2}$} & mean & 332.34 & 318.42 & 268.23 & 241.01 \\
\hline & std & 29.42 & 30.87 & 35.42 & $\underline{57.40}$ \\
\hline \multirow{2}{*}{$f_{3}$} & mean & 439.52 & 439.52 & 264.42 & 164.21 \\
\hline & std & $1.59 e-13$ & $1.01 e-05$ & $\underline{18.87}$ & 15.57 \\
\hline \multirow{2}{*}{$f_{4}$} & mean & 278.74 & $\underline{237.56}$ & $\underline{259.70}$ & 206.20 \\
\hline & std & 26.38 & $\underline{22.04}$ & $\underline{18.24}$ & 35.71 \\
\hline \multirow{2}{*}{$f_{5}$} & mean & 158.16 & $\underline{148.85}$ & 148.52 & 133.07 \\
\hline & std & 11.10 & $\underline{13.50}$ & $\underline{11.26}$ & $\underline{18.17}$ \\
\hline \multirow{2}{*}{$f_{6}$} & mean & 336.00 & $\underline{307.96}$ & $\underline{324.77}$ & $\underline{309.15}$ \\
\hline & std & 22.35 & $\underline{27.63}$ & $\underline{19.34}$ & $\underline{26.64}$ \\
\hline \multirow{2}{*}{$f_{7}$} & mean & 9.16 & $\underline{8.04}$ & 9.41 & 10.29 \\
\hline & std & 1.39 & 4.84 & 5.61 & 3.91 \\
\hline \multirow{2}{*}{$f_{8}$} & mean & 48.66 & $\underline{45.24}$ & 46.27 & $\underline{38.75}$ \\
\hline & std & 2.42 & $\underline{7.87}$ & 5.70 & $\underline{10.63}$ \\
\hline \multirow{2}{*}{$f_{9}$} & mean & 13.07 & $\underline{12.42}$ & 12.84 & 15.64 \\
\hline & std & 1.62 & $\underline{1.27}$ & 1.71 & 2.90 \\
\hline \multirow{2}{*}{$f_{10}$} & mean & 111.91 & 119.44 & 113.46 & 110.60 \\
\hline & std & 11.94 & 8.15 & 14.87 & 2.23 \\
\hline \multirow{2}{*}{$f_{11}$} & mean & 53.95 & $\underline{46.40}$ & $\underline{51.13}$ & $\underline{45.91}$ \\
\hline & std & 2.11 & $\underline{1.13}$ & $\underline{6.12}$ & $\underline{3.66}$ \\
\hline \multirow{2}{*}{$f_{12}$} & mean & 99.50 & $\overline{85.89}$ & $\overline{99.62}$ & $\overline{87.09}$ \\
\hline & std & 6.49 & $\underline{5.53}$ & 4.79 & $\underline{6.51}$ \\
\hline \multirow{2}{*}{$f_{13}$} & mean & 129.64 & $\underline{117.20}$ & 121.67 & $\underline{94.70}$ \\
\hline & std & $4.06 e-14$ & 15.10 & 12.28 & 7.35 \\
\hline \multirow{2}{*}{$f_{14}$} & mean & 102.98 & 72.97 & 96.67 & 91.69 \\
\hline & std & 6.09 & 9.02 & 3.77 & 6.43 \\
\hline \multirow{2}{*}{$f_{15}$} & mean & 9.94 & $\underline{8.47}$ & 9.10 & 8.20 \\
\hline & std & 0.58 & $\underline{1.17}$ & 1.65 & $\underline{1.51}$ \\
\hline \multirow{2}{*}{$f_{16}$} & mean & 90.15 & 86.58 & $\underline{76.50}$ & $\underline{61.23}$ \\
\hline & std & 7.58 & 7.40 & $\underline{3.21}$ & $\underline{6.19}$ \\
\hline \multirow{2}{*}{$f_{17}$} & mean & 52.78 & 61.23 & $\underline{43.59}$ & 50.05 \\
\hline & std & 2.54 & 4.17 & $\underline{4.65}$ & 3.52 \\
\hline \multirow{2}{*}{$f_{18}$} & mean & 51.64 & 49.34 & $\underline{42.64}$ & 48.85 \\
\hline & std & 8.37 & 7.03 & $\underline{7.59}$ & 9.90 \\
\hline \multirow{2}{*}{$f_{19}$} & mean & 1.51 & $\underline{1.11}$ & $\underline{1.23}$ & $\underline{0.83}$ \\
\hline & std & 1.31 & $\underline{1.16}$ & $\underline{1.16}$ & $\overline{0.28}$ \\
\hline \multirow{2}{*}{$f_{20}$} & mean & 48.48 & 48.23 & $\underline{39.52}$ & 45.85 \\
\hline & std & 0.10 & 0.83 & 3.03 & 2.81 \\
\hline
\end{tabular}




\section{Acknowledgements}

T. Zhang acknowledges partial support from National Science Foundation (NSF) grant No. 1200566. Moreover, M. Georgiopoulos acknowledges partial support from NSF grants Nos. 0806931, 0963146, and 1161228. Finally, G. C. Anagnostopoulos acknowledges partial support from NSF grant No. 1263011. Any opinions, findings, and conclusions or recommendations expressed in this material are those of the authors and do not necessarily reflect the views of the NSF.

\section{References}

[1] W. Armstrong, P. Christen, E. McCreath, and A.P. Rendell. Dynamic algorithm selection using reinforcement learning. AIDM '06, pages 18-25, 2006.

[2] Yoav Benjamini and Daniel Yekutieli. The control of the false discovery rate in multiple testing under dependency. The Annals of Statistics, 29:919-1188, 2001.

[3] Mauro Birattari, Zhi Yuan, Prasanna Balaprakash, and Thomas Stützle. F-race and iterated F-race: An overview. Technical report, IRIDIA, 2011.

[4] J. Brest, S. Greiner, B. Boskovic, M. Mernik, and V. Zumer. Self-adapting control parameters in differential evolution: A comparative study on numerical benchmark problems. IEEE Trans. on Evol. Comput., 10:646-657, 2006.

[5] Vincent A. Cicirello and Stephen F. Smith. Heuristic selection for stochastic search optimization: Modeling solution quality by extreme value theory. Lect. Notes in Compu. Sc., 3258:197-211, 2004.

[6] Vincent A. Cicirello and Stephen F. Smith. The max K-Armed bandit: A new model of eploration applied to search heuristic selection. In 20th Natl. Conf. on Artificial Intelligence, pages 1355-1361, 2005.

[7] Stuart Coles. An Introduction to Statistical Modeling of Extreme Values. Springer, 2001.

[8] P. de Zea Bermudez and Samuel Kotz. Paremeter estimation of the generalized pareto distribution part I. J. Stat. Plan. Inference, 140:1353-1373, 2010.

[9] M. Gagliolo and J. Schmidhuber. Algorithm selection as a bandit problem with unbounded losses. In LION 4 Proc. of the Learning and Intelligent Optimization Conf., pages 82-96, 2010.

[10] M. Gallagher and B. Yuan. A general-purpose tunable landscape generator. IEEE Trans. on Evol. Comput., 10:590-603, 2006.

[11] N. Hansen, S.D. Müller, and P. Koumoutsakos. Reducing the time complexity of the derandomized evolution strategy with covariance matrix adaptation (CMA-ES). Evol. Comput., 11:77-112, 2003.

[12] Jürg Hüsler, Pedro Cruz, Andreia Hall, and Carlos M. Fonseca. On optimization and extreme value theory. Meth. And Comput. In Appl. Probab., 5:183-195, 2003.

[13] Michail G. Lagoudakis and Michael L. Littman. Algorithm selection using reinforcement learning. In Proc. of the 17th Int. Conf. on Machine Learning, pages 511-518, 2000.

[14] Oded Maron and Andrew Moore. Hoeffding races: Accelerating model selection search for classification and function approximation. Advances in Neural Information Processing Systems, 6:59-66, 1994.

[15] V. Mnih, C. Szepesvári, and Jean yves Audibert. Empirical bernstein stopping. In Proc. of the 25th Int. Conf. on Machine Learning, 2008.

[16] Andrew W. Moore. Efficient algorithms for minimizing cross validation error. In Proc. of the 11th Int. Conf. on Machine Learning, pages 190-198, 1994. 
[17] F. Peng, K. Tang, G. Chen, and X. Yao. Population-based algorithm portfolios for numerical optimization. IEEE Trans. on Evol. Comput., 14:782-800, 2010.

[18] A. K. Qin, V. L. Huang, and P. N. Suganthan. Differential evolution algorithm with strategy adaptation for global numerical optimization. IEEE Trans. on Evol. Comput., 3:398-417, 2009.

[19] Carl Scarrott and Anna MacDonald. A review of extreme value threshold estimation and uncertainty quantification. Statistical Journal, 10:33-60, 2012.

[20] F. van den Bergh and A.P. Engelbrecht. A cooperative approach to particle swarm optimization. IEEE Trans. on Evol. Comput., 8:225- 239, 2004.

[21] J. Vrugt, B. Robinson, and J. Hyman. Self-adaptive multimethod search for global optimization in real-parameter spaces. IEEE Trans. on Evol. Comput., 13:243-259, 2009.

[22] Chunlin Wang. A new hybrid estimation method for the generalized pareto distribution. Master's thesis, University of Calgary, 2011.

[23] Shiu Yin Yuen, Chi Kin Chow, and Xin Zhang. Which algorithm should I choose at any point of the search: an evolutionary portfolio approach. In GECCO '13 Proceeding of the Genet. and Evol. Comput. Conf., pages 567-574, 2013.

[24] J. Zhang and A. C. Sanderson. JADE: Adaptive differential evolution with optional external archive. IEEE Trans. on Evol. Comput., 13:945-958, 2009.

[25] Tiantian Zhang, Giselle Borrero, and Michael Georgiopoulos. A Winner-Take-All methodology: Finding the best evolutionary algorithm for the global optimization of functions. In GECCO'12 Proc. of the Genet. and Evol. Comput. Conf. Companion, pages 1515-1516, 2012.

[26] Tiantian Zhang, Michael Georgiopoulos, and Georgios C. Anagnostopoulos. S-Race: A multi-objective racing algorithm. In GECCO'13 Proc. of the Genet. and Evol. Comput. Conf., pages 1565-1572, 2013.

[27] Q. Zheng, Y. Fan, Z. Shi, and Y. Wang. Adaptive inertia weight particle swarm optimization. In ICAISC'06 Proc. of Artificial Intelligence and Soft Computing, volume 4029, pages 450-459, 2006. 